\title{
Effects of pore-channel ordering on mechanical properties of anodic aluminium oxide nano-honeycombs
}

\author{
K.Y. Ng $^{*}$ and A.H.W. Ngan \\ Department of Mechanical Engineering, The University of Hong Kong, Pokfulam Road, \\ Hong Kong, PR China \\ * Correspondence Author (Email:kycng@hku.hk)
}

\begin{abstract}
The mechanical properties of anodic aluminum oxide (AAO) nano-honeycombs with different spatial ordering of pore-channels are investigated by nanoindentation. The pore-channel ordering is systematically varied by carefully conditioning the orientation of the aluminum for anodization. The results indicate that the strength of AAO structures increases significantly with the regularity of their pore-channel arrangement, whereas the elastic modulus is less sensitive to the pore-channel regularity.
\end{abstract}

Keywords: anodic aluminium oxide; grain orientation; plastic deformation; nanoindentation. 
Anodic aluminum oxide (AAO) produced using electrochemical oxidation of aluminum exhibits a unique nano-honeycomb structure in which straight pore-channels of diameters often less than 100nm may extend over lengths exceeding $100 \mu \mathrm{m}$ [1-3]. AAO is considered as an important material in nanotechnology fabrication, and is used as template for the fabrication of other nanostructures such as nano-tube and nano-wire arrays [4-9], as well as in making functional devices such as electroosmotic pumps [10] and gas sensors [11]. Two recent developments should also help accelerate the industrial exploitation of this material: firstly, Lee et al. [12] developed a "hard anodization" approach to fabricate AAO many times faster the conventional method, and secondly, we [13] developed a method of obtaining AAO structures with selective ordering of the pore-channels homogeneously over $\mathrm{mm}^{2}$-to- $\mathrm{cm}^{2}$ areas. We discovered that, in the two-step anodization method [1] in oxalic acid, AAO films grown on top of large [001]-oriented recrystallized aluminum grains exhibited an arrangement of the pore channels close to ideal close-packing in a domain structure, whereas that in films grown on [101] grains did not show any resemblance to close packing at all, and other orientations of the substrate Al yielded intermediate pore ordering [13]. This method of carefully conditioning the orientation of the aluminum substrate enables the ordering of the pore-channels in the AAO nanohoneycomb structure to be actively controlled over unprecedented large areas. This is important for the fabrication of structures and devices with consistent performance, and also allows systematic investigation of properties of AAO versus the spatial ordering of the pore-channels. 
In this paper, we report an investigation on the mechanical properties of AAO with varying pore-channel ordering. Although the mechanical behavior of macroscopic honeycomb structures has received a lot of interest in the mechanics community [14-19], the mechanical properties of AAO nanohoneycombs are much less studied. We recently investigated the nanoindentation [20] as well as uniaxial compressive [21] behavior of AAO with non-specific pore-channel ordering. In this paper, we focus on elucidating the effects of pore-channel ordering on the nanoindentation behavior of AAO.

To establish the conditions of producing AAO structures with homogeneous pore-channel ordering over large areas, the starting material was chosen to be a $3 \mathrm{~mm}$ thick aluminum slab with purity $>99.9999 \%$ [13]. This slab was subjected to a series of thermomechanical treatments with the purpose of achieving large recrystallized grains in the mm-to-cm size range. The procedure included first cold rolling the $\mathrm{Al}$ slab by $10 \%$ thickness reduction, followed by a first annealing step at $450^{\circ} \mathrm{C}$ for 2 hours, and then a second annealing step at $550^{\circ} \mathrm{C}$ for two days, in a vacuum better than $10^{-7}$ torr. The heat-treated slab was then cut into discs of 1 inch diameter by electric discharge machining, and these were then annealed again to remove any residual stresses left. To produce the necessary smooth surface for the later electro-anodization process, the discs were first mechanically polished by 240-, 600-, 1200-, 2400-, and 4000-grit SiC sand papers in succession, and then electo-polished in an electrolyte of $20 \%$ perchloric acid in ethanol at $20 \mathrm{~V}$ dc and $-10^{\circ} \mathrm{C}$. The pre-treated $\mathrm{Al}$ discs were then subjected to anodization in a $0.3 \mathrm{M}$ oxalic acid solution at $40 \mathrm{~V} \mathrm{DC}$ in a constant temperature environment of $17 \pm 0.1^{\circ} \mathrm{C}$ provided by an electronic feed-back water bath. The two-step anodization 
method [1] was employed to further improve the quality of the AAO films [13]. Here, an initial layer of AAO was formed in a first anodization step which lasted typically for about 15 hours. This layer was then dissolved away by a phosphochromic acid solution (1.5wt $\% \mathrm{H}_{2} \mathrm{CrO}_{4}$ in $6 \mathrm{wt} \% \mathrm{H}_{3} \mathrm{PO}_{4}$ ) leaving behind more regular dimpled pits on the metal substrate which acted as seeds for the growth of more regular pore channels in a second anodization step, which typically lasted for 10 hours. As in our previous work [13], the as-prepared AAO structures were found to develop pore-channel ordering highly specific to the orientation of the underlying Al grain. Scanning electron microscopy (SEM) examination showed that the pore-channel ordering was uniform over the entire area of each underlying $\mathrm{Al}$ grain, which was from a few $\mathrm{mm}^{2}$ to a few $\mathrm{cm}^{2}$ in size, and the pore-channel ordering was different over differently orientated Al grains [13].

Nanoindentation was carried out on the AAO structures supported by their $\mathrm{Al}$ substrates in a Nanohardness Tester (CSEM, Switzerland) with a Berkovich diamond tip. An exponential loading profile was used in which the load $P$ varied with time $t$ according to $P=P_{0} \exp (\kappa t)$, and this should correspond to a constant-strain-rate condition [22] with the indentation strain rate equal to $\kappa / 2$. The constant $\kappa$ was set to be $0.007675 \mathrm{~s}^{-1}$ for the load ramp, and at the maximum load which was $50 \mathrm{mN}$, the load was held for 1 min, followed by unloading to $10 \%$ of the maximum load at a rate of $50 \mathrm{mN} / \mathrm{min}$. The load was held at the $10 \%$ value for another $1 \mathrm{~min}$ in order to estimate the thermal drift rate during the testing period, followed by complete unload. At least 15 indention tests were performed on a single grain, and altogether more than 30 grains were studied. 
The AAO structures before and after nanoindentation were examined in a LEO 1530 FEG-SEM, as well as in a Tecnai 20 transmission electron microscope (TEM). Longitudinal sections of the AAO structures parallel to the pore-channels for both SEM and TEM were made by focused-ion-beam (FIB) milling in an FEI Quanta 3D FIB system.

Figs. 1(a-c) show the plan-view SEM micrographs of three AAO structures with decreasing degrees of spatial ordering of the pore-channels from (a) to (c). By dissolving away the AAO film by a phosphochromic acid solution in order to expose the underlying Al for orientation measurement by electron backscattered diffraction, the orientations of the substrate $\mathrm{Al}$ grains in these three situations were determined to be [106], [125] and [011] respectively. In a recent report [13], over one hundred grains were analyzed this way, and a similar relation was observed consistently: high ordering of AAO pore-channels, similar to that in Fig. 1(a), was observed on [001]-oriented grains, and poor ordering of pore-channels, similar to Fig. 1(c), was observed on [011]-oriented grains. The highly ordered case in Fig. 1(a) exhibits close packing of the pore-channels in domains of a few microns wide. On the contrary, the poorly ordered case in Fig. 1(c) does not show any resemblance to close-pack arrangement. To quantify the pore-channel ordering, the relative positions of the pores as seen in plan-view SEM images were identified by an image-processing software ImageJ (ver.1.42). This software was used to search the pixel coordinates of the centroids of the individual pores, which were then used to calculate the 2-D radial distribution function (RDF) of the pore pattern. The RDF is defined as $[d F(r) / d r] /(2 \pi r)$, where $F(r)$ is the fraction of pore pairs $i-j$ with 
center-to-center inter-pore distances $r_{i j}$ smaller than or equal to $r$. The corresponding RDFs of the three structures are shown as insets in Fig. 1(a-c). The peaks in such an RDF correspond to the regularity of the pore positions, whereas the background joining the troughs of the peaks is due to the irregularity of the pores. Thus, a measure of the pore ordering is the area sandwiched between the RDF and the smooth background, and is termed the "RDF peak intensity" [13]. In the following, the RDF peak intensity is also expressed as a percentage normalized by the maximum value encountered in our study. The RDF peak intensity and the normalized value (blanketed) was determined to be $3.013 \times 10^{-4}(95 \%), 1.623 \times 10^{-4}(54 \%)$ and $7.913 \times 10^{-5}(26 \%)$ for the three cases in Fig. $1(\mathrm{a}-\mathrm{c})$ respectively, and these indicators are consistent with the ordering as seen from the SEM images. Fig. 1(d,e) shows the longitudinal-section TEM images of the highly ordered AAO structure grown on top of the [106]-oriented Al grain, and the poorly ordered structure on top of the [011]-oriented grain, respectively. The contrast in these two images is due to absorption by the amorphous alumina channel walls. It can be seen that the pore-channels in Fig. 1(d) are straight and parallel, while those in Fig. 1(e) have a high tendency to tilt and merge.

Fig. 2(a) shows the representative nanoindentation load-displacement curves of AAO structures with three different ordering. Serrations are observed, but in terms of these, there is no significant difference between the different pore ordering. The most obvious effect is that the highly ordered structure (with RDF peak intensity $=94.5 \%$ ) is significant harder than the poorly ordered structure. Figs. 2(c,d) summarize the hardness and elastic modulus measured using the Oliver-Pharr method [23] from all the AAO 
structures investigated, versus their RDF peak intensity values. The hardness of the AAO in Fig. 2(c) clearly rises as the pore-channel ordering increases. However, the measured elastic moduli of the AAO structures in Fig. 2(d) do not show a clear trend with the pore-channel ordering.

Fig. 2(b) shows plan-view SEM images of the nano-indent arrays made on the AAO structures with different pore ordering. It is interesting to see that while the indents in the highly ordered case on the left are equilateral triangular in shape, those in the poorly ordered case on the right have triangular shapes which are not equilateral but elongated in different directions. For example, indent A in the poorly ordered case elongates along the 7:30 o'clock direction, while indent B elongates along the 11:00 o'clock direction. The inconsistency in the indent shape in the poorly ordered case is unlikely due to the undulation of the AAO surface, which is estimated to be 1 to 2 microns at maximum. It is more likely a manifestation of the random arrangement of the pore channels in this case.

The high-magnification plan-view SEM images in Fig. 3 show the details of the deformation in single indents. The tri-star shape of the plan-view structure is consistent with our earlier observation [20] that two different deformation modes, one for a region closer to the indent center and the other for a region further away from it, occurred. The deformation closer to the indent center corresponds to severe compaction of the porous AAO structure, whereas that further away from the indent center is milder and corresponds to tilting of the pore-channel walls instead of full collapse of the structure. 
The cross-sectional SEM images in Fig. 4 confirm these two deformation modes of full compaction near the indent center and channel-wall tilting near the indent rim region. Again, as was observed previously [20], a sharp elasto-plastic boundary exists in all the indents shown.

In a series of papers [24-27], Chung et al. studied the strength of three-dimensional networks and found that the correlation length is the predominant parameter that determines the failure stress under uniaxial stress. In the present work, the total area of the pores in the AAO structures was found to decrease as the pore ordering decreased. As an indication, the three AAO structures in Fig. 1(a-c) had average pore sizes of $75 \pm 5 \mathrm{~nm}, 69 \pm 10 \mathrm{~nm}$ and $59 \pm 20 \mathrm{~nm}$ respectively, and average wall thicknesses of $15 \pm 5 \mathrm{~nm}, 18 \pm 10 \mathrm{~nm}$ and $25 \pm 15 \mathrm{~nm}$ respectively. In other words, more disordered AAO structures possess higher solid volume compared to ordered ones, and they might be expected to be stronger under load. However, the measured hardness was found to exhibit the opposite trend: for the three structures in Fig. 1(a-c), the hardness was $470 \pm 20 \mathrm{MPa}, 422 \pm 15 \mathrm{MPa}$ and $353 \pm 30 \mathrm{MPa}$ respectively, i.e. more disordered structures were weaker. The results here suggest that pore-channel ordering has a significant influence on the strength of the AAO structures, and this influence is not describable as an effect of a mean correlation length alone. Instead, the results show that the spread (i.e. variance) of the correlation length is a significant factor, namely, more regular arrangement of the pore-channels results in a stronger structure, and vice versa. This is not surprising, as in a more disordered structure, the stress distribution due to a given external load situation will be more non-uniform, with greater chance of 
occurrence of extreme stress values at locations of stress raisers. Since failure should start at locations with extreme stress values, a disordered structure should be weaker than an ordered structure. One of us performed finite-element simulations on elastic networks with varying ordering [18], and found that the statistical distribution of the internal forces indeed is a strong signature of the structure's regularity. Yield locus analysis also shows that, when subjected to the same overall load, irregular structures are weaker than regular structures [19]. The present hardness results in Fig. 2(c) illustrate this phenomenon very well. As discussed before, the inconsistency of the indent shape in the irregular AAO structure in Fig. 3 is also a likely consequence of the structural irregularity. The more pronounced tilting, branching and merging of the pore-channels in a more poorly ordered structure (see Fig. 1(e) and Fig. 4) are likely to result in large local stress concentrators, and when one of these is interacted upon by an indentation field, more severe deformation will occur there, leading to more anisotropic deformation and elongation of the indent shape along one direction. On the other hand, the elastic modulus (Fig. 2(d)) is less affected by such stress concentration effects. The present results illustrate that the spatial ordering of the pore-channels is an important factor governing the mechanical strength of AAO. With the present technique to control the pore-channel ordering by substrate conditioning during anodization, other properties of AAO structures can be investigated systematically as a function of their pore-channel ordering.

In summary, this work illustrate a novel method, which involves carefully conditioning the orientation of the aluminum substrate for anodization, to produce AAO structures with tunable and homogeneous pore-channel ordering over $\mathrm{mm}^{2}$-to- $\mathrm{cm}^{2}$ areas. 
Nanoindentation tests on such AAO structures with controlled pore-channel ordering indicate that their strength increases significantly with the regularity of their pore-channel arrangement.

The authors thank Prof. B.J. Duggan for kindly providing the aluminum slabs used in this study. The work described in this paper was supported by grants from the Research Grants Council (Project No. HKU7159/10E ), as well as from the University Grants Committee (Project No. SEG-HKU06) of the Hong Kong Special Administration Region, P.R. China. 


\section{Figure captions:}

Fig.1 (a-c): Plan-view SEM morphology of (a) highly ordered AAO on [106] Al substrate (RDF index: $3.013 \times 10^{-4}(95 \%)$ ); (b) intermediate ordered AAO on [125] Al substrate (RDF index: $1.623 \times 10^{-4}(54 \%)$ ); and (c) poorly ordered AAO on [011] Al substrate (RDF index:7.913×10-5(26\%)). (d-e): TEM micrographs showing the longitudinal sectional pore-channel morphology of (d) a highly ordered and (e) a poorly ordered AAO structures.

Fig.2 (a): Nanoindentation load-displacement curves of AAO structures with three different ordering. (b): SEM images of the nano-indent arrays made on the AAO structures with different pore ordering. (c-d): Oliver-Pharr hardness (c) and elastic modulus (d) versus the RDF peak intensity values of the AAO structures studied.

Fig.3 The high-magnification plan-view SEM images of three AAO structures of different pore-channel ordering

Fig.4 The cross-sectional SEM images of the three AAO structures in Fig. 3 


\section{References}

[1] H. Masuda, K. Fukuda, Science 268 (1995)1466.

[2] H. Masuda, F. Hasegwa, S. Ono, J Electrochem Soc. 144(1997)L127.

[3] H. Masuda, H. Yamada, M. Satoh, H. Asoh, M. Naka, T. Tamamura, Appl Phys Lett. 71(1997)2770.

[4] W.C. Hu, D.W. Gong, Z. Chen, L.M. Yuan, K. Saito, C.A. Grimes, P. Kichambare, Appl. Phys. Lett. 79(2001)3083.

[5] Y. Zhang, G.H. Li, Y.C. Wu, B. Zhang, W.H. Song, L. Zhang, Adv. Mater 14(2002)1227.

[6] M. J. Zheng, L. D. Zhang, X. Y. Zhang, J. Zhang, G. H. Li, Chem. Phys. Lett. 334(2001)298.

[7] M. S. Sander, A. L. Prieto, R. Gronsky, T. Sands, A. M. Stacy, Adv. Mater 14(2002)665.

[8] M. S. Sander, R. Gronsky, T. Sands, A. M. Stacy, Chem. Mater. 15(2003)335.

[9] Y. Li, G. S. Cheng, L. D. Zhang, J. Mater. Res. 15(2000)2305.

[10] S. K. Vajandar et al., Nanotechnology 18(2007)

[11] O. K. Varghese, D. W. Gong, W. R. Dreschel, K. G. Ong, C. A. Grimes, Sens. Actuators, B 94, (2003) 27. 
[12] W. Lee, R. Ji, U. Gosele, K. Nielsch, Nat. Mater. 5(2006)741.

[13] K.Y. Ng, A.H.W. Ngan, Chem. Mater., accepted.

[14] T. C. Triantafillou, J. Zhang, T. L. Shercliff, L. J. Gibson, M.F. Ashby, Int J Mech Sci. 31(1989)665.

[15] I. G. Masters, K.E. Evans, Compos Struct. 35(1996)403.

[16] L. J. Gibson, M. F. Ashby, J. Zhang, T. C. Triantafillou, Int J Mech Sci. 31(1989)635.

[17] C. Chen, T. J. Lu, N.A. Fleck, J Mech Phys Solid. 47(1999)2235.

[18] A.H.W. Ngan, Proc Roy Soc Lond. A461(2005)433.

[19] A.H.W. Ngan, Proc Roy Soc Lond. A461(2005)1423.

[20] K.Y. Ng, Y. Lin, A.H.W Ngan. Acta Mater. 57(2009)2710.

[21] K. Y. Ng, Y. Lin, A. H. W. Ngan, J Mech Phys Solids. 59 (2011) 251.

[22] B.N. Lucas, W.C. Oliver, Metallurgical and Materials Transactions. 30A (1999)601. 
[23] W.C. Oliver and G.M. Pharr, J. Mater Res. 7(1992)1564.

[24] J. W. Chung, A. Roos, and J. Th. M. De Hosson Phys. Rev. B 54 (1996) 15094.

[25] J. W. Chung, J. Th. M. De Hosson,* and E. van der Giessen Phys. Rev. B 64 (2001) 064202.

[26] J. W. Chung, J. Th. M. De Hosson, and E. van der Giessen Phys. Rev. B 65(2002) 094104.

[27] J. W. Chung and J. Th. M. De Hosson Phys. Rev. B 66 (2002) 064206. 
Figures

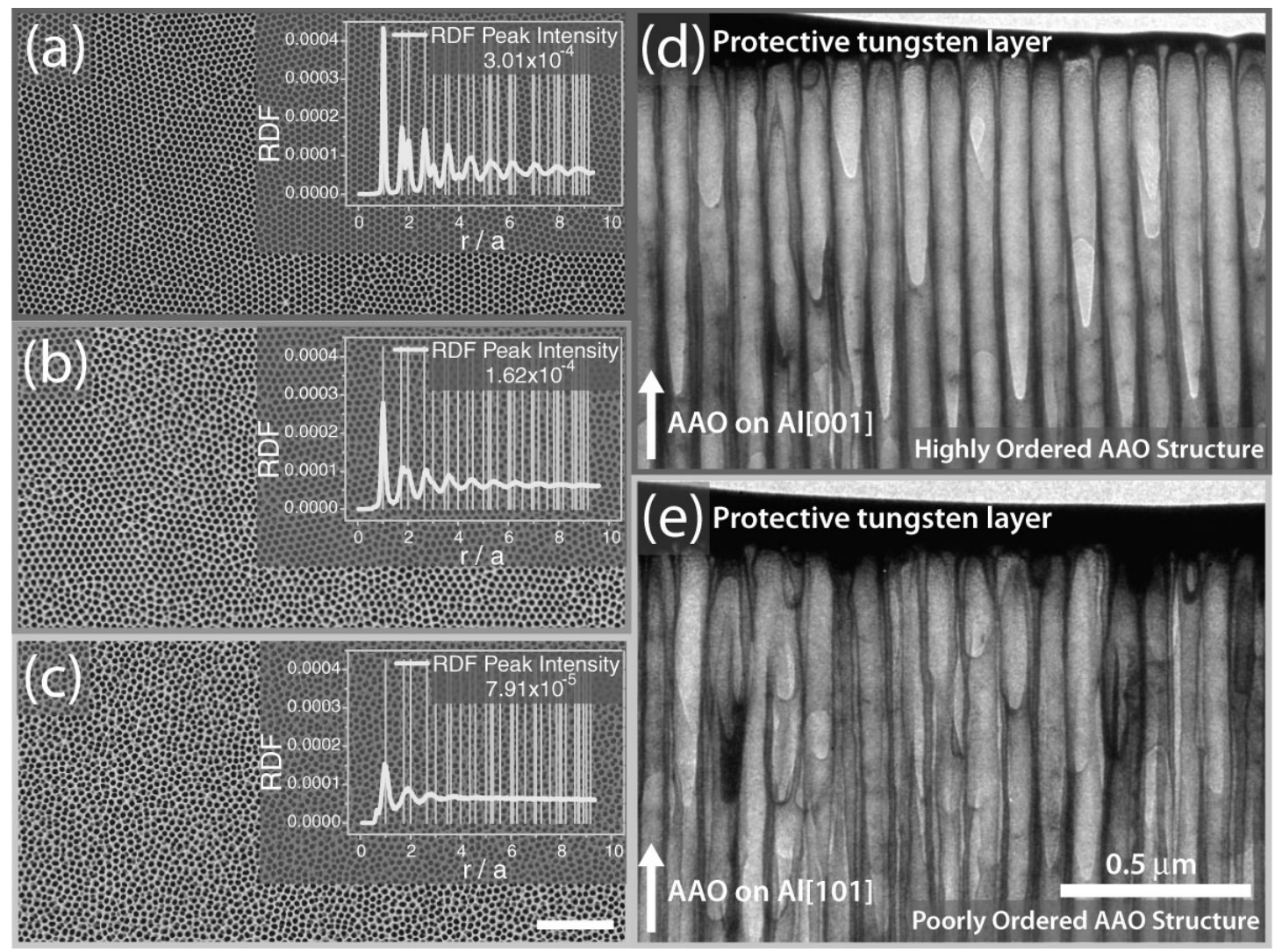

Fig.1 

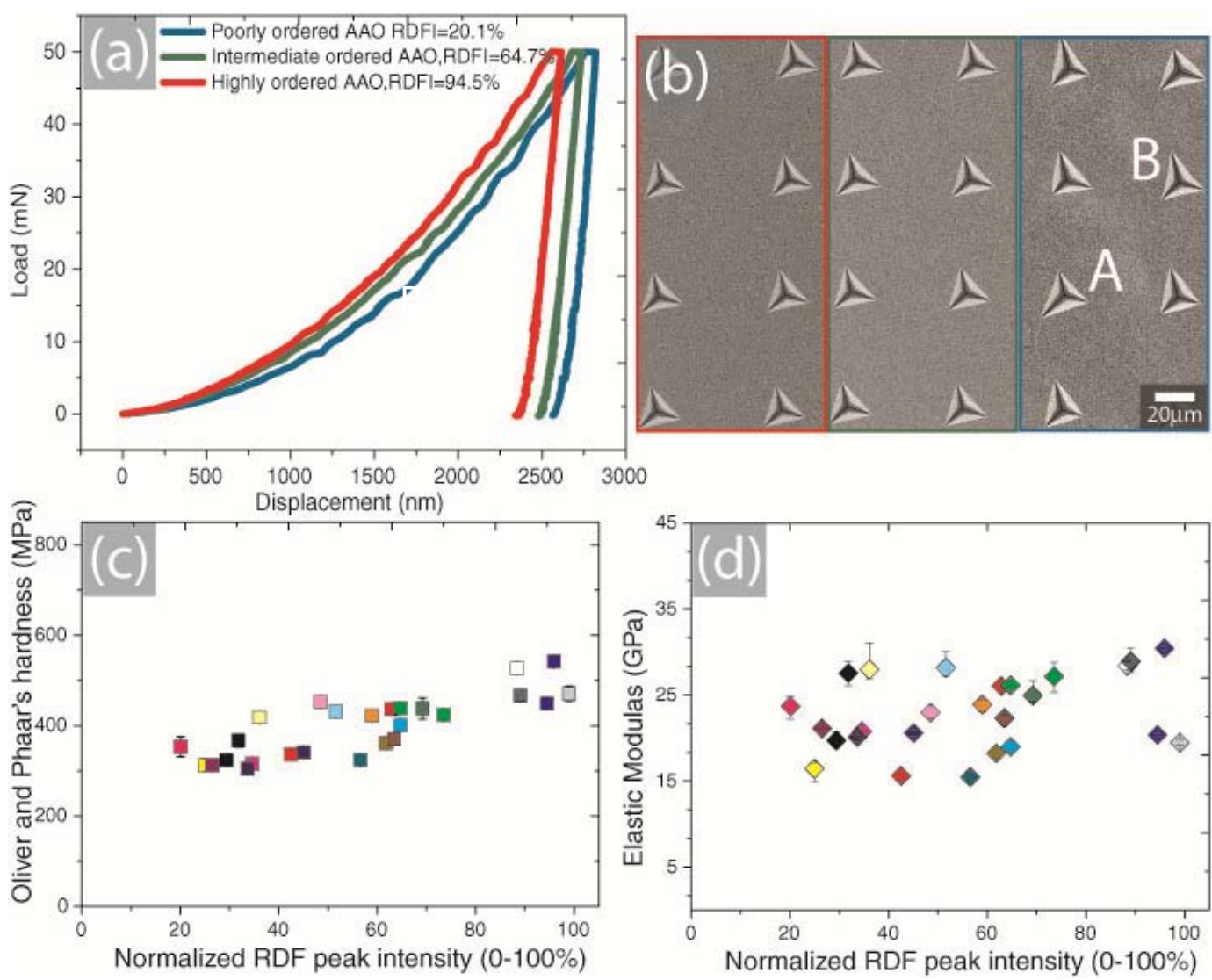

Fig. 2 


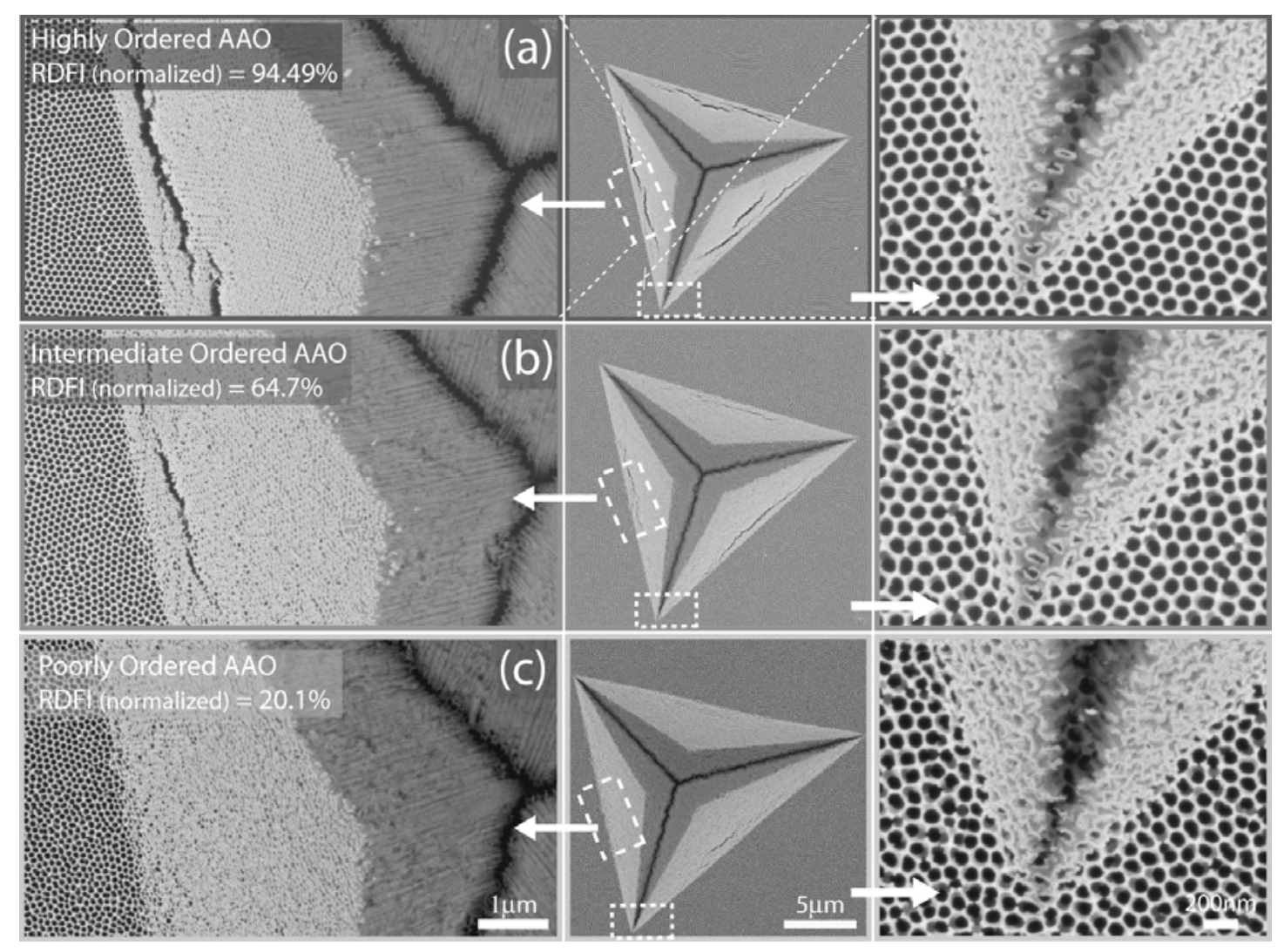

Fig. 3

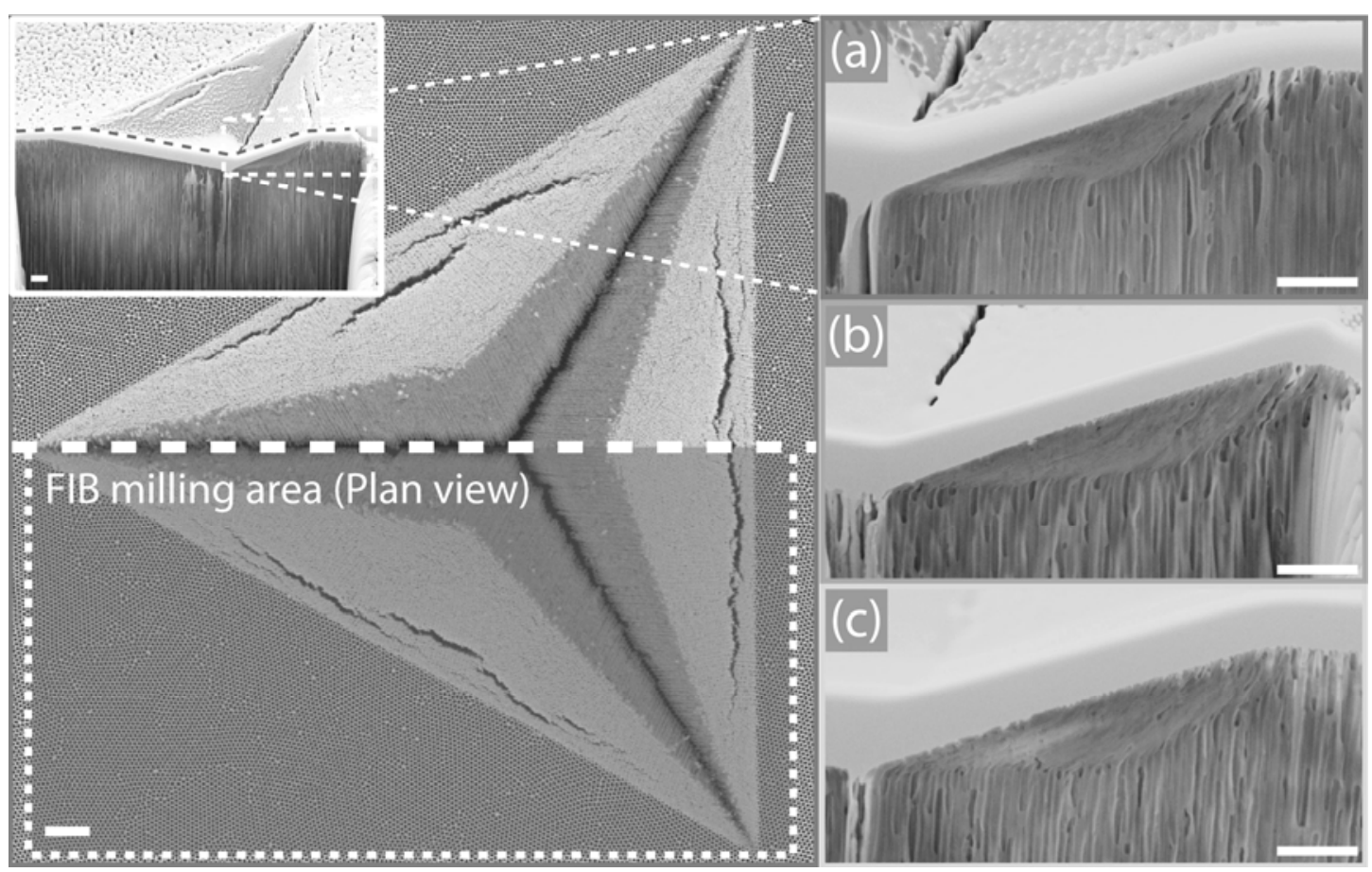

Fig. 4 
